\title{
A Universal Method to Control over-time Alterations of Respiratory Sinus Arrhythmia in Short and Long Signals
}

\author{
Iga Grzegorczyk ${ }^{1}$, Jan Gierałtowski ${ }^{1}$, Paweł Krzesiński ${ }^{2}$, Jan Żebrowski ${ }^{1}$ \\ ${ }^{1}$ Faculty of Physics, Warsaw University of Technology, Warsaw, Poland \\ ${ }^{2}$ Department of Cardiology and Internal Diseases. Military Institute of Medicine
}

\begin{abstract}
One of the ways allowing to control the performance of the Autonomic Nervous System (ANS) is monitoring the cardiorespiratory coupling. It shows how the two most important systems in the human body act to function efficiently. The effect that is believed to show these dependencies is Respiratory Sinus Arrhythmia (RSA), which causes accelerations of heart rate during inspiration and decelerates the rhythm during passive act of expiration. There are many methods to analyse the occurrence of RSA, from the basic peak-to-valley method, to more complicated ones as e.g. spectral coherence. But all of them have flaws like omitting samples or giving only a single value for the entire analysed period.

In this paper, we present a simple, numeric method, which enables the analysis of the changes in the strength of RSA over the time. Our solution is called Cardiorespiratory Coupling Assessment (CCA) and calculates the value of RSA in consecutive, one-minute long windows as the median of the differences of $R R$ intervals detected during expiration and inspiration in each window.

The data necessary for the CCA method to work properly includes ECG and the respiratory signal from the chest belt measured simultaneously. In our research, we first analysed the performance of the method on synthetically generated data to verify its performance. Than we compared short 5 minute recordings obtained during spontaneous and controlled breathing and then analysed 4 hours long signals extracted from nighttime recordings.

The results show that the CCA method allows to measure accurately the changes in strength of RSA over the time in both short and long signals. Moreover, the results indicate that the level of the RSA strength is larger in recordings from spontaneous breathing phase than from the controlled breathing.
\end{abstract}

\section{Introduction}

The autonomic nervous system is responsible for the proper functioning of the human body. Among other things, it controls both the cardiovascular and respiratory systems. One effect that allows to monitor the relation between the two systems is the Respiratory Sinus Arrhythmia (RSA). It is responsible for improving gas exchange in the lungs and the efficacy of blood circulation in the body [1]. It manifests itself by shortening the intervals between consecutive heart beats during inspiration and elongating them during expiration phase of breathing. The most commonly used methods for the measurement of this phenomenon are the peak-to-valley method and spectral analysis, both of which are able to determine whether RSA occurred in a particular signal. However, there is no golden standard to measure the RSA effect over the time or of showing the strength of this effect.

This is the reason why we propose a new method Cardiorespiratory Coupling Assessment (CCA) - allowing to follow the changes in RSA in the time domain. The CCA method is easy to understand from the physiological point of view. Moreover, it is suitable for the analysis of short as well as long time series, which will be shown below.

\section{Data}

The dataset analysed in this paper consisted of simultaneously registered: ECG signals from lead II and respiratory recordings from a chest belt.

The short signals were of 5 minutes length and were registered during the controlled breathing test (CBT) in supine position and the rest period before it. The sampling frequency was $200 \mathrm{~Hz}$. The study population included 70 healthy subjects.

The long signals were 4 hours long each and were extracted from all night polysomnographic recordings. 
The sampling of the ECG signals was $200 \mathrm{~Hz}$ and the respiratory signals from the chest belt were originally sampled at $100 \mathrm{~Hz}$ and were resampled to $200 \mathrm{~Hz}$ to match the ECG signals. The subjects examined during polysomnography were also healthy.

\section{Method}

The Cardiorespiratory Coupling Assessment (CCA) method proposed in this paper is a mathematical tool with two main features. First, it is simple in its structure. Second and foremost, it is easily understandable for physicians because of the clear, physiological reasoning behind it. To assess the RSA effect with this method the ECG and the respiratory signal should be measured simultaneously.

\subsection{Pre-processing}

To make the analysis possible, both ECG and respiratory, signals have to be properly prepared and their main features have to be extracted.

ECG: The first important step in preparation of the ECG signal is to filter out all frequencies except the range $1-40 \mathrm{~Hz}$ to avoid unphysiological noise e.g. of electrical origin. Than the locations of the $\mathrm{R}$ peaks have to be found, which is obtained with algorithm described in $[2,3]$. Having the locations of the R peaks, RR intervals are calculated and then they are assigned to the location of the second $\mathrm{R}$ peak from which each RR interval was calculated.

Respiratory signal: The process of respiratory signal analysis in this case begins with detrending performed with a non-causal mean filter of window length equal to 1 s. Next, the minima and maxima of the respiratory curve are annotated. Then, the signal is divided into inspiration and expiration phases. The first of them is recognized by the abruptly increasing slopes of the respiratory curve, and the latter as the decreasing slopes and together with the plateaus that often occur between the consecutive inspirations.

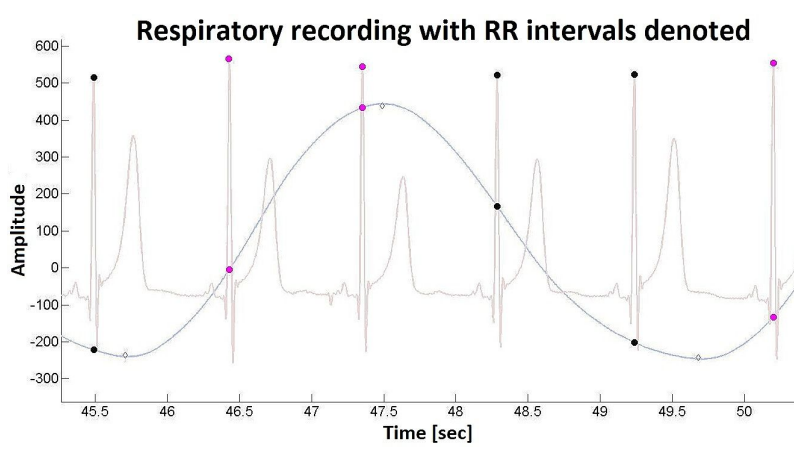

Figure 1 Example showing how the RR intervals are distributed on the respiratory curve according to inspiration and expiration phase. The location of the second $\mathrm{R}$ peak decides to which respiratory phase the $\mathrm{RR}$ interval is assigned.

\subsection{Cardiorespiratory Coupling Assessment}

Next, we create a moving window of 60 seconds length. It is moved with a step of 1 sample. At each location of the window, the RR intervals inside it are assigned into one of two groups: inspiratory or expiratory. Than, the median of RR intervals in each group is calculated and assigned to the end of the window. The window moves one step and the process is repeated. This way, we obtain a curve showing the changes in the median of RR intervals during the inspiratory phase and another curve for the expiratory phase. The final step is to subtract these two curves. The obtained result is CCA measure. Now we can create a plot showing continuous changes of the RSA effect in the time domain (comp. Fig 2, 3 and 4).

\section{Results}

The plots below show how does the CCA measure perform for signals of different length and structure. Figure 2a shows that when a subject is breathing regularly in a relatively slow pace (approximately 7 breathes per minute) the CCA measure becomes large, what means that the RSA effect is strong. Because of high parasympathetic activity in such situations the obtained result was expected. 

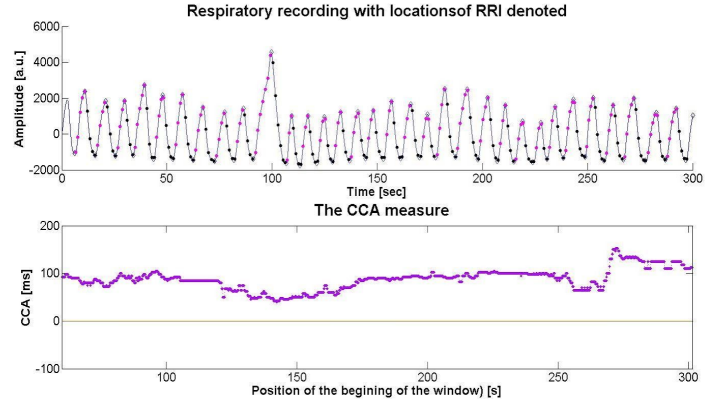

Figure 2 The high CCA values obtained for the calmly breathing subject - visible RSA effect. Signal registered during controlled breathing test.

On the other hand, when a subject is taking fast, shallow breaths the RSA effect should not occur, as the parasympathetic activity is decreased. Such situation is presented on the Figure 3.
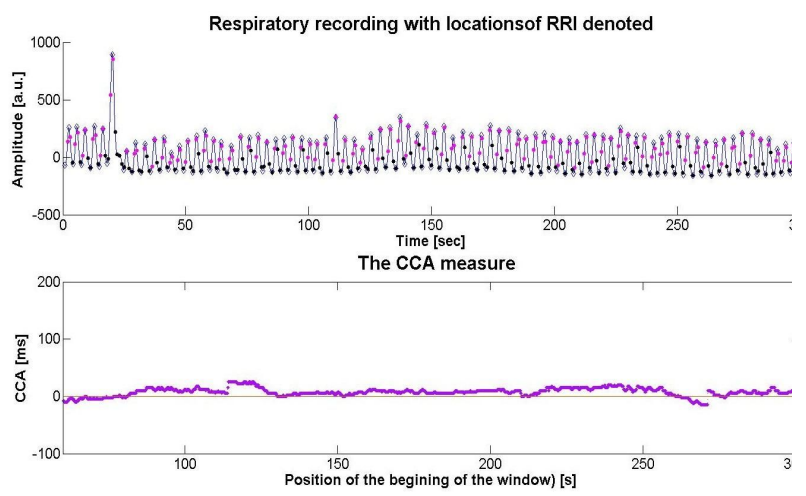

Figure 3 The CCA measure oscillates around zero as the patient takes fast, shallow breaths - no RSA effect. Signal registered during the rest phase (spontaneous breathing).

The last example shows that the CCA method can be also applied to signals of long duration (Figure 4). In Figure 4 there is only the CCA curve, as the respiratory signal of 4 hours length was unreadable in this time scale. The plot below shows, that the RSA effect is definitely not a constant parameter and changes over the time. It can be seen that the CCA measure sometimes attains values below zero. The interpretation of this is not quite clear at this moment.

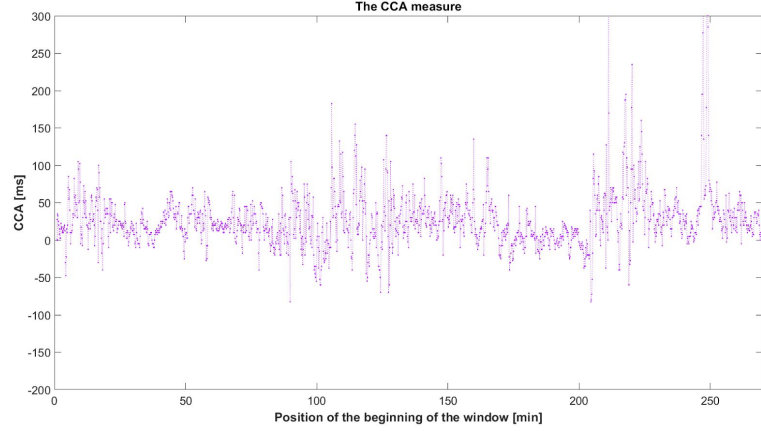

Figure 4 The results of CCA method applied to a 4 hour long signal registered during polysomnography.

\section{Discussion and conclusions}

The proposed method was created to allow the analysis of changes in the RSA effect in time domain. The main requirements were for it to be mathematically correct and easily understandable for physicians. In this paper, we showed that the CCA method indicates that modifications in strength of the RSA effect occur with the time and that the method is suitable for the analysis of both short and long time series. A paper with more details of tests performed on the method is in the preparation.

The analysis were performed only for the respiratory signals registered with a chest belt. In the future, we plan to adapt the method to the analysis of data obtained from nasal airflow sensors, which will require taking into account the difference in phase of the respiratory curve obtained by these two measurement methods. It would broaden the applicability of the method to different data types.

\section{References}

[1] Hayano, Junichiro, et al. "Respiratory sinus arrhythmia." Circulation 94.4 (1996): 842-847.

[2] Behar Joachim, Jonhson Alistair, Clifford Gari D., Oster Julien A Comparison of Single Channel Foetal ECG Extraction Methods. Annals of Biomedical Engineering. 42(6), 1340-53. 2014.

[3] Johnson, A. E., Behar, J., Andreotti, F., Clifford, G. D., \& Oster, J. (2014, September). R-peak estimation using multimodal lead switching. In Computing in Cardiology Conference (CinC), 2014 (pp. 281-284). IEEE.

Address for correspondence:

Iga Grzegorczyk,

Warsaw University of Technology, Faculty of Physics

Koszykowa 75 00-662 Warsaw, Poland

igagrze@gmail.com 
Page 4 